\title{
On selecting emotional outcomes in a rehabilitation program for persons with traumatic brain injury in Brazil
}

\author{
Ana Paula Almeida de Pereira \\ Universidade Federal do Paraná, Curitiba, PR, Brazil
}

\begin{abstract}
Traumatic brain injury (TBI) is an important health problem in Brazil. It frequently results in a variety of sequelae, including cognitive, behavioral, and physical impairments that are frequently related to difficulties in community reintegration and successful rehabilitation outcomes. The literature shows that several variables are involved in positive outcomes, including neuropsychological deficits, services received, and social support. Brazil has a paucity of rehabilitation programs for persons with TBI. Intervention outcomes are underreported, and culturally adapted rehabilitation strategies are rarely found. The present study describes a rehabilitation program, presents emotional factors as an important outcome, and discusses a case that illustrates the major points debated. Keywords: brain injury, emotional outcome, rehabilitation, program evaluation, neuropsychological assessment, outcome assessment.
\end{abstract}

Received 21 February 2011; received in revised form 30 August 2011; accepted 8 September 2011. Available on line 10 October 2011

\section{Introduction}

Traumatic brain injury (TBI) occurs when sudden trauma damages the brain. Traumatic brain injury is an important health problem worldwide. According to the Centers for Disease Control and Prevention in the United States, at least 1.4 million people sustained TBI and 50,000 of these individuals die each year (Centers for Disease Control and Prevention, 2005). The most frequent causes of TBI are falls, motor vehicle accidents and violence. Worldwide findings are similar and studies from several countries have reported that working-age males are at risk for TBI.

Traumatic brain injury frequently results in a variety of sequelae including cognitive, behavioral and physical impairments (Parker, 1990). Among the most commonly reported cognitive deficits are difficulties learning new information because of attention problems and memory limitations, restricted judgment skills and a diminished ability to plan and act accordingly. Behaviorally, Prigatano (2005) pointed out several symptoms such as apathy, emotional lability, paranoid reactions, reduced tolerance to frustration and diminished impulse control. Silver, Kramer, Greenwald and Weissman (2001) studied the relationship between brain injury and psychiatric

Correspondence regarding this article should be directed to: Ana Paula Almeida de Pereira, Departamento de Psicologia, Universidade Federal do Paraná, Praça Santos Andrade, 50, Curitiba, Paraná, CEP: 80.060-240 Brazil. Phone: +55-41-3310-2625. Fax: +55-41-3310-2641. E-mail: anapaula_depereira@yahoo.com disorders and found a higher probability of psychiatric disorders in the group of patients with head injury. Blurred or double vision, problems with motor speed and coordination, seizures and fatigue are commonly reported physical complaints.

In the last two decades, efforts have been made in Brazil to address the neuropsychological sequelae of TBI and to promote rehabilitation (Abrisqueta-Gomez \& Santos, 2006). Nevertheless, effective strategies to address deficits that affect persons with TBI are still being developed. Brazil has a paucity of rehabilitation programs for persons with TBI. Intervention outcomes are underreported and culturally adapted rehabilitation strategies are rarely found.

The present study briefly describes an outpatient rehabilitation program, Projeto CEREI (Centro de Estudos em Reabilitação e Inclusão), reports the main challenges encountered in establishing program outcomes, proposes a rationale for selecting emotional aspects as one important program outcome and briefly discusses a case study that illustrates the role of emotional factors during rehabilitation.

\section{Description of CEREI program}

CEREI was created in 2007, linked to the Department of Psychology at the Universidade Federal do Parana, to study the rehabilitation process of adults with disabilities caused by brain injury. As a service offered at a public university and related to student training, a limited number of patients are admitted 
each year. Patients are referred by their neurologists at least 2 months after their injury. Their ages range from 18 to 55 years. The CEREI program consists of four major services provided during a period of 8 months: initial assessment (i.e., interviews, questionnaires and neuropsychological battery), psychotherapy, cognitive rehabilitation and family support. The following section presents each service and briefly describes their purpose.

\section{Initial assessment}

All individuals who enter the program undergo an initial assessment. In selecting initial assessment procedures, two major criteria are used: inclusion of wellknown standardized assessment tools that can facilitate future comparative studies and instruments with good ecological validity that can report a patient's potential and limitations while developing the individual rehabilitation plan. The assessment battery covers eight domains: Intelligence, Attention/Concentration, Learning and Memory, Executive Function, Social Skills, Emotional Status, Functional Status and Quality of Life.

After the initial assessment, a rehabilitation plan is developed based on the assessment results and on the priorities of the patients and their families. Interventions in at least three areas are included in the plan: Emotional Aspects, Cognitive and Functional Factors and Community Participation. The CEREI program adopted a holistic approach to neuropsychological rehabilitation (Ben-Yishay \& Daniels-Zide, 2000).

\section{Cognitive rehabilitation}

Traditionally, the rehabilitation of neuropsychological disorders has focused on two major goals: restoration and compensation. Restoration aims to rebuild lost abilities. However, restitution would be a realistic goal if damage to a particular brain system is incomplete so that the remaining neurons within a neural circuit have the potential to reconnect (Glisky, 2004). Compensation for neuropsychological deficits refers to the utilization of equipment and environmental cues to augment existing abilities (Skeed \& Edwards, 2001). There is still much debate about how to implement and train patients to use such aids (Sohlberg \& Mateer, 2001).

Wilson (2002) listed the main predictors of good use of compensation in patients: age (the younger the better), severity of impairment (people with very severe impairment compensate less well), specificity of deficit (the more specific the better) and premorbid use of strategies. These factors are considered while selecting rehabilitation interventions.

The CEREI program focuses on compensatory rehabilitation strategies and adapted the strategies described by Sohlberg and Mateer (2001) and High, Sander, Struchen and Hart (2005) to the Brazilian environment. Clients have a weekly appointment at the clinic. The 60-min cognitive rehabilitation session is divided into two parts. In the first part of the session, problems performing routine activities are identified and explored; later, strategies that might help clients solve these problems are developed and recorded in a notebook or on tape so the clients can refer to these strategies at home. The second part consists of structured exercises (e.g., computer games and paper and pencil tasks) that address specific cognitive problems. The strategies to perform them are explained, a sample exercise is completed and other similar exercises are assigned to be performed at home. The cognitive rehabilitation process is discussed with the family during monthly meetings.

\section{Family support}

After TBI, families have to find a new balance to cope with the demands of taking care of a family member with special needs. The rehabilitation process can be a burden to primary family caregivers (Wade et al., 2002). Family needs also change as clients move along the rehabilitation process continuum (Moules \& Chandler, 1999; Rotondi, Sinkule, Balzer, Harris, \& Moldovan, 2007). Several studies showed that families influence the achievement of different rehabilitation goals such as behavioral and academic achievement (Mazzucchi et al., 2000; Yeates et al., 2002).

Families are an integral part of the rehabilitation process. Programs that include interventions that provide support to families report their role in medication monitoring, case management and relapse prevention. Especially in Brazilian culture, families are the major support system for persons with TBI and frequently the major care providers.

Sohlberg and Mateer (2001) described a family collaborative model and showed that clinician-family partnerships might facilitate rehabilitation goals and help shape the intervention that will work in the client's context. However, the authors recognized that families also need support during the rehabilitation process. Such an approach tends to enhance rehabilitation results. Therefore, a "significant other" is invited to participate in cognitive rehabilitation and a monthly interview with the families is organized as part of the rehabilitation process.

\section{Psychotherapy after TBI}

The complex emotional factors involved in psychotherapy during rehabilitation are well known. Considering the limited intervention time period available for the program and need to establish major variables that could be assessed and become part of the program outcome evaluation protocol, the CEREI program adopted two criteria to guide the selection of variables: variables should be constructs coherent with a theoretical framework and variables should be directly related to the rehabilitation process. These guidelines address a common critique of rehabilitation 
strategies: the frequent lack of a theoretical framework that integrates the intervention and justifies clinical decisions (Wilson, 1991). In an effort to create an evidence-based practice with consistent theoretical support, CEREI proposed an existential therapeutic approach in which patients in the program have weekly 50-min psychotherapy sessions.

The emotional aspects involved in the rehabilitation process of people who have experienced TBI have been studied by numerous researchers (Flaherty-Craig, Barrett, \& Eslinger, 2005). Many persons with brain injury have consistently exhibited problems readapting to work and school and maintaining social interactions because of emotional difficulties. Research results appear to agree about the importance of providing cognitive rehabilitation and therapeutic interventions during the rehabilitation process (Miller, 1993; Parker, 1990; Prigatano, 2005).

Parker (1990) described confusion, anxiety, anger and identity problems as common reactions after the traumatic event. Frequently, people with brain injury are told that they have fully recovered from their physical symptoms and that they are able to return to their regular activities. Some patients feel that they have not recovered, with at least four emotional reactions: confusion, anxiety, anger and difficulties recognizing one's identity. People with TBI usually show anxiety that cannot be linked to any specific situation but pervades every part of his world experience. Consequently, the person may seem physically able to resume previous activities, but individuals frequently report the contrary emotionally.

In the CEREI program, four major psychological problems are assessed and given special attention because they tend to be commonly reported within this group: self-awareness, anxiety, anger and depression. The present article proposes that these aspects should be included as part of a program's evaluation factors.

\section{Challenges establishing program outcomes in Brazil}

Traditionally, cognitive function and returning to work are the most commonly emphasized aspects of a rehabilitation plan. Nevertheless, restricting program evaluation to these factors disregards important cultural and economic aspects of the Brazilian context and neglects another desirable outcome, specifically adequate emotional perception and expression.

There are only a few neuropsychological instruments adapted to Portuguese and available in Brazil and most of them require that the examinee has several years of education. Both facts considerably limit the use of neuropsychological instruments during the rehabilitation program assessment phase. Moreover, the lack of Brazilian studies that have investigated the ecological validity (Naldone \& Stringer, 2001) of the published instruments represents an important problem that was tentatively overcome by the CEREI program that combines quantitative and qualitative procedures (e.g., semi-structured assessment interview with patient and significant other). Such qualitative procedures allowed a variety of factors to be reported. Relying only on the results of neuropsychological instruments would limit program evaluation.

Returning to work remains a major goal in rehabilitation, which usually indicates that the patient has recovered his/her premorbid level of cognitive and social skills. Employment may also promote a better financial situation. Brazil, however, has important barriers to achieving such a goal. Most persons with moderate or severe TBI qualify for disability benefits. This benefit is given only if the person is considered incapable of working. Thus, attempts to attain full-time employment among people with disabilities is not common because they do not want to risk losing their life-long benefit. A more flexible law is necessary in which efforts to reenter the work force, even in a part-time temporary job, are valued.

To motivate persons with disability to return to work, a policy that provides support to promote better training and a higher educational level in this group could also be effective. Such a policy would enable persons with disabilities to earn higher wages that could compensate for the risk of losing stable benefits.

In summary, other outcome variables are needed to provide a more accurate measure of the efficacy of interventions. The holistic model adopted by CEREI considers other variables as important outcomes, including life meaning, sense of peace, social activities, capacity for joy and intimacy (Wilson, 2003). These aspects are addressed by CEREI's psychotherapeutic and family support intervention. The patient's emotional perception and expression are included as relevant outcome variables in the CEREI program. As mentioned above, emotional factors are highly correlated with successful rehabilitation but are usually neglected as program outcome variables. The present article focuses on the emotional aspects that arise during the rehabilitation process, how to address them in individual rehabilitation plans and their importance as a rehabilitation outcome.

\section{Existential-phenomenological approach to address emotional variables}

Considering the emotional aspects commonly found after TBI, CEREI elected to use a brief psychotherapeutic intervention based on the Existential-Phenomenological approach, which presents a theoretical framework for understanding some of the most frequently observed emotional reactions after TBI and suggests intervention strategies to deal with them. 


\section{Self-awareness after TBI}

Impaired self-awareness is a recognized component of TBI sequelae that limits clients' engagement in rehabilitation and successful adoption of appropriate goals (Prigatano \& Schacter, 1991). The literature agrees on three major points: (1) self-awareness requires the integration of several cognitive and affective functions, (2) lesions of the prefrontal cortex are usually related to the presence of deficits in self-awareness and (3) deficits in self-awareness are potential obstacles to successful rehabilitation. Fleming, Tooth, Connell and Strong (2002) compared the levels of self-awareness in rehabilitation clients with TBI and spinal cord injury (SCI) during the period of transition from hospital to home. Their findings suggested that self-awareness was more impaired in participants with TBI. Moreover, the researchers proposed that during the initial period of rehabilitation, both clients and their families can experience difficulties realizing significant ongoing sequelae. They also defend rehabilitation procedures as a way to increase the awareness of deficits (Port, Willmott, \& Charlton, 2002).

Awareness is a multi-faceted construct that encompasses physical awareness, environmental awareness and social awareness. The scientific literature on the topic agrees that the concept of awareness is complex. The present study focuses on the awareness of physical status and mental abilities and adopts the term self-awareness.

Sohlberg and Mateer (2001) reviewed three broad approaches to self-awareness intervention: individual awareness-enhancing program, caregiver training and education and procedural training and environmental support. The individual awareness-enhancing approach is recommended for clients who have sufficient cognitive capacity to integrate information and experience but not for clients with dense, global unawareness with cognitive impairments. CEREI's clientele consists of persons with mild to moderate brain injury; therefore, a combination of strategies was adopted: educational techniques (used during family orientation) and experiential exercises (individual awareness-enhancing techniques used during psychotherapy).

The Patient Competency Rating Scale (PCRS; Prigatano, 1986) has been broadly used as a measure of self-awareness in which the examinee and a significant other complete a paper and pencil scale (Sherer, Harts, \& Nick, 2003). PCRS covers the areas of functional ability (e.g., How much of a problem do I have preparing my own meals?), interpersonal skills (e.g., How much of a problem do I have recognizing that something I say or do has upset someone else?) and emotional status (e.g., How much of a problem do I have keeping myself from being depressed?). This instrument is used during the initial and posttreatment assessments.
Although different strategies and various instruments are used to assess self-awareness, the use of more than one measurement strategy is recommended as a way to address the complexity of this construct. Thus, in addition to the PCRS form, the CEREI assessment interview with patients and their families also includes items that investigate this variable. The assessment of self-awareness is an important part of developing a rehabilitation plan. Several studies have used changes in self-awareness to evaluate rehabilitation outcomes and the present study also used this domain as part of the CEREI program's outcome assessment.

\section{Anxiety after TBI}

Sohlberg and Mateer (2001) stated that after brain injury anxiety could be manifested as nervousness, insecurity, or fear. Anxiety might also arise as a consequence of the perception of reduced functional ability, increased experience of failure and concern about the future.

King (1997) reviewed the literature on the various aspects involved in the period following mild head trauma, specifically delineating the post-concussion syndrome (PCS). This syndrome is characterized by four factors: cognitive factor, somatic factor, affective factor and sensory factor. King analyzed emotional factors during recovery as (1) anxiety and distress concerning PCS and its treatment and (2) posttraumatic stress disorder as a result of the traumatic incidents that cause head injuries (King, 1997, p. 176).

Furthermore, anxiety caused by prolonged organic recovery is as likely to occur as anxiety related to reestablishing a routine after a period of hospitalization (King, 1997). Anxiety is an expected emotional reaction to the situations a person with brain injury must face, regardless of whether cognitive deficits (such as concentration problems and memory loss) are present. In summary, anxiety has always been reported as a major factor during rehabilitation (King, 1997; Miller, 1993; Prigatano, 2005). Therefore, an assessment of anxiety (i.e., the Beck Anxiety Inventory-Portuguese version) is used and therapeutic procedures to deal with it are included as routine procedures at CEREI.

\section{Depression after TBI}

After the innumerable interventions in the cognitive and somatic spheres during the rehabilitation process, a person with TBI might feel different about his abilities and limitations. The traumatic event could be a disrupting factor in the previous psychological balance. This disruption might mean that the person was confronted with an identity crisis situation when values and goals had to be reviewed.

Freed (2002) exemplified the barriers confronted by persons with brain injury in reintegrating their ego and establishing meaningful relationships by reporting two of her cases. Freed (2002) concluded that relationships 
that were built before the traumatic event tended to dissolve after the changes began to appear because of deep feelings of loss shared by both the client and the significant other and escalating anxiety.

The literature also indicates that some adaptation of the assessment and therapeutic techniques is needed to carefully interpret ideas and behaviors, discriminating which characteristics are related to organic problems and psychogenic problems and understanding cognitive limitations and emotional psychodynamics (Freed, 2002; Prigatano, 2005; Sohlberg \& Mateer, 2001). Potentially confusing symptoms of brain damage reflect the neurogenic syndrome but can be confounding factors when diagnosing depression: lack of initiative, impaired emotional expressiveness, lowered crying threshold and fatigue.

In summary, although there are still some difficulties assessing depression after TBI, the early detection of depression in this group is encouraged because depression is commonly reported as one barrier to successful rehabilitation in long-term outcome studies (Dawson, Schwartz, Winocur, \& Stuss, 2007; Draper, Pondsford, \& Schonberger, 2007). CEREI uses questions in the initial assessment interview and the Beck Depression Inventory (BDI)-Portuguese version to assess depressive symptoms.

\section{Anger outbursts}

Another emotional aspect frequently reported as part of the psychological changes after TBI is anger outbursts. Miller (1993) devoted a chapter of his book to this topic. Considerable evidence indicates that this behavior is caused by brain dysfunction linked to different areas of the brain. The frontal lobes and temporal lobe limbic structures are examples of structures often injured, which are connected to such emotional reactions.

Freed (2002) delineated a different explanation in her case studies. She related the reactions both to previous aspects of the patient's personality and to the frustration some specific environmental or relationship problem could trigger, leaving the organic explanation as secondary.

Anger can be considered a part of the human existential condition. Every person must negotiate life while resisting, acknowledging, and assuming possibilities and limitations. Therefore, the problem would consist of unexamined, repetitive, or sustained anger. Anger could be trigged by the perception of helplessness and can become a beneficial emotion in the person's growth. Anger is not a pleasurable experience but can become rewarding as long as some thought is given to it. In such an approach, the TBI clients' angry reactions (i.e., retained or expressed) should be explored to facilitate the comprehension of the internal and external contexts involved. If helplessness increases anger, then how is the client empowered? What would be the procedures during rehabilitation that facilitate the negotiation of the life course? If emotion signifies the relationship one has with the world, then it would be therapeutic to understand this relationship, negotiate it and transform it. Psychotherapy may be an important tool for facilitating this process after TBI.

\section{Framework for understanding emotional reactions}

Existential psychology suggests that emotions have a meaning and that their meaning is closely connected to the way the person perceives the world. The established relationship guides the personal reality. With this approach, each emotion can signify various aspects of the experience of being in the world with a sensitive body on different occasions.

May (1983) developed a series of works that adopted the existential perspective in the field of psychology. $\mathrm{He}$ defined the world as a structure of meaningful relationships in which a person exists and in the design of which he participates. Consequently, in existential theory, man and world are dynamically understood and man participates in and designs the world at the same time. May (1983) described simultaneous and interrelated aspects of the world that delineate the existence of each individual as a being in the world. Moreover, in this perspective, the future opens up man's possibility of becoming who man chooses to be, which may increase anxiety. Responsibility and freedom are two basic concepts of existential psychology and they are also key elements in the rehabilitation process because they allow self-determination.

May (1967) envisioned different problems that might arise in the relationship described, which created psychopathology. One of these problems is impotence. A person can develop impotence when he does not believe he can make a difference or act meaningfully in the world. According to the author, impotence might become anxiety, anxiety might become regression and apathy, regression and apathy might become hostility and hostility might become the alienation of the person from others.

At this point, an initial hypothesis can be formulated to articulate in a meaningful way the emotional reactions described as present after TBI. After identifying the emotional reactions commonly observed during the rehabilitation process of persons with TBI and comparing them with the aforementioned sequence of emotional reactions (May, 1967), similar phenomena can be hypothesized in the rehabilitation of persons with TBI. If the injury suddenly evoked a period of intense cognitive deficits or even physical limitations, then the person probably had the feeling (even for a little while) of being a stranger in a dangerous and inexplicable world.

The confrontation of this situation could create the perception of impotence that triggers the following sequence of reactions: anxiety, apathy, hostility and isolation. This sequence is obviously not a temporal, stage-like process but rather a turbulent one in which 
the different modes of being in the world are affected. Additionally, emotions in this situation would represent, from an existential point of view, this turbulent being in a strange world: the environment is puzzling, the significant others are unfamiliar and the person in the mirror struggles to be recognized.

In summary, the present section presented the major emotional aspects addressed during CEREI's psychotherapeutic intervention and the theoretical approach adopted as they provide the foundation for the assessment of emotional outcomes of the rehabilitation process.

\section{Case study}

The case study reported here sought to carry out an in-depth investigation of the rehabilitation experiences of a person with severe TBI. Initially, the results from the initial and final assessments are briefly presented and the emotional outcomes (i.e., levels of self-awareness, anxiety, depression and anger) are then discussed.

The participant, BO, was 52 years old and had completed high school at the time of the initial assessment (A1). His intelligence, measured by the Wechsler Adult Intelligence Scale (WAIS), was average (Table 1), but he and his family presented several complaints about his memory, executive skills, emotional reactions and social abilities during the first interview. His disability was a result from a closed-head injury in a car accident 6 years before his enrollment in the program. Before the accident, he worked at a family business as a financial manager. After the accident, he retired and received state benefits. His accident injured his frontal and temporal lobes and he lost most of the vision of his left eye. He was in a coma for 3 months and later had 2 months of physical therapy and speech and language therapy.

The initial and final assessments consisted of an interview and the administration of several instruments: WAIS-III, Delis-Kaplan Executive Function System subtests (D-KEFS), Rey Auditory Verbal Learning Test (RAVLT), Rey Complex Figure Test (RCFT), PCRS, Beck Anxiety Scale (BAI), BDI and WHO Quality of Life-brief inventory (WHO-QOL). The final assessment was administered 1 year after the patient's enrollment in the program.

As shown in Table 1, during the initial assessment, measures of verbal memory (RAVLT), executive function (D-KEFS-trail making subtest and PCRS), emotional reactions and quality of life presented poor results (Mitrushina, Boone, Razani, \& D'Elia, 2005). His individual rehabilitation plan targeted (1) building memory and compensatory organizational and planning strategies (e.g., diary, calendar, agenda, cellular phone alarms), (2) addressing the reported emotional problems (especially depressive symptoms, anger outbursts and self-awareness) and (3) enhancing social interactions. Importantly, returning to work was not an issue for BO because he had other income sources and his priorities were related to his cognitive and emotional situation.

Cognitive rehabilitation initially focused on introducing the use of an agenda and a calendar because BO complained about his difficulties remembering his appointments and organizing his routine. After the second month of treatment, planning skills were taught to help develop a yearly plan that led him to look at his future possibilities instead of constantly complaining about his losses after the accident. These abilities diminished the number of situations in which BO felt frustrated and inadequate.

Initially, BO's wife participated in monthly interviews in the program. She had three major complaints about her husband: irritability, apathy and mood swings. During the fourth month of treatment, BO had a serious quarrel with his daughter. This event trigged his wife to ask for a divorce. From that moment on, she did not participate in any of the program's activities. Two family interviews were done with BO's daughters during the second semester of the year-long program. They reported several problems in their relationship with their father caused by his communication difficulties and irritability.

The divorce from his wife deeply affected BO and motivated him to effectively participate in the psychotherapeutic process. After that event, he realized that he was responsible for his current situation and lessened his blame on others for his problems. He also agreed to take medication previously prescribed by his psychiatrist (i.e., mood stabilizer and sleeping pills). His emotional problems were then explored and his behaviors were analyzed by discussing the conditions that trigged anxiety and anger and how he could deal with them. He used his diary to describe these events and "work through" possible solutions.

BO gradually engaged in different learning activities, such as internet skills and foreign language classes, that allowed him to meet new people with similar interests. The fact that the new acquaintances did not know his abilities before the accident contributed to his feeling of being more at ease with interpersonal interactions. Another interesting fact was that his primary family (brothers and sisters) became closer to him and he would frequently be invited to family events.

After 1 year, a second assessment was done. The cognitive tests did not show significant improvement, but important differences were found with regard to emotional reaction scales and quality of life measures. Moreover, his report during the final interview revealed that he considered that the treatment was successful because he was living independently and achieved a more stable emotional and social life. He said, "I came out of the box because of the program," so emotional well-being was apparently an important outcome in this case. 
Table 1. Participant's scores on the initial (A1) and final (A2) asssessments

\begin{tabular}{|c|c|c|}
\hline Scale & A1 & $\mathrm{A} 2$ \\
\hline WAIS-III & $\mathrm{SS}^{*}$ & $\mathrm{SS}^{*}$ \\
\hline Similarities & 13 & 13 \\
\hline Arithmetic & 13 & 14 \\
\hline Digit Span & 14 & 12 \\
\hline Information & 16 & 15 \\
\hline L-N Sequence & 11 & 14 \\
\hline Picture Completion & 10 & 12 \\
\hline Digit Symbol & 9 & 9 \\
\hline Block Design & 11 & 11 \\
\hline Matrix Reasoning & 12 & 15 \\
\hline Symbol Search & 10 & 9 \\
\hline D-KEFS & $\mathrm{SS}^{*}$ & SS* \\
\hline Tower & 7 & 8 \\
\hline Trial (Condition 4) & 1 & 1 \\
\hline Color-Word (Condition 3) & 1 & 5 \\
\hline Color-Word (Condition 4) & 6 & 8 \\
\hline Design Fluency & 7 & 6 \\
\hline RAVLT & RS* & RS* \\
\hline Trial 5 & 9 & 9 \\
\hline Trial 6 & 4 & 5 \\
\hline Trial 7 & 4 & 5 \\
\hline BAI & 14 & 16 \\
\hline BDI & 19 & 11 \\
\hline PCRS & 88 & 109 \\
\hline WHO-QOL & 69 & 78 \\
\hline
\end{tabular}

*SS, scaled scores and RS, raw scores

\section{Discussion}

One purpose of this case presentation is to point out that emotional well-being tends to be a more appropriate outcome to be considered across different groups with TBI, such as older persons with severe injury, whereas traditional outcome variables, such as returning to work and cognitive performance, appear to be a specific goal for groups of younger adults.
Although the clients' affective reactions may be similar in many circumstances, their meaning, from an existential perspective, is particular to a range of psychological factors related to the way the person relates to the world. Finally, it should be stressed that where there is meaningful life, there is selfhood.

From this perspective, rehabilitation reflects reconstruction of the meaning of life for the person with TBI. The present article argues that addressing existential questions and adopting a phenomenological method in 
the psychotherapeutic process of the TBI client provides a coherent theoretical framework for understanding the emotional problems usually observed in people with TBI and points to important rehabilitation goals.

The domain of rehabilitation can benefit from the Existential-Phenomenological approach because, as discussed above, persons with TBI clearly mention existential issues during the rehabilitation process. Although few studies in the rehabilitation field have adopted the Existential-Phenomenological theoretical foundation (Nadell, 1991; Ososkie, 1998), the present discussion might provide theoretical contributions and useful parameters to guide interventions and techniques to address the emotional aspects of the rehabilitation process.

In conclusion, the literature shows that a person's successful rehabilitation is related to his level of selfawareness and the process of reconstituting a sense of self. Such achievements help to cope with anxiety, depressive affect and anger outbursts. Thus, the assessment of these variables and a close follow-up of their interconnections during psychotherapy is a crucial part of the CEREI rehabilitation program.

\section{References}

Abrisqueta-Gomez, J., \& Santos, F.H. (2006). Reabilitação neuropsicológica: da teoria a prática. Sao Paulo: Artes Médicas.

Ben-Yishay, Y., \& Daniels-Zide, E. (2000). Examined lives: outcomes after holistic rehabilitation. Rehabilitation Psychology, 45, 112-129.

Centers for Disease Control and Prevention (2005). Facts about traumatic brain injury. Retrieved from: http://www.cdc.gov/ Migrated.Content/Fac.Sheet/Freeform_Fact_Sheet_(General)/ FactsAbout TBI

Draper, K., Ponsford, J., \& Schönberger, M. (2007). Psychosocial and emotional outcomes 10 years following traumatic brain injury. Journal of Head Trauma Rehabilitation, 22, 278-287.

Dawson, D.R., Schwartz, M.L., Winocur G., \& Stuss, D.T. (2007). Return to productivity following traumatic brain injury: cognitive, psychological, physical, spiritual and environmental correlates. Disability and Rehabilitation, 29, 301-313.

Flaherty-Craig, C.V., Barrett, A.M., \& Eslinger, P.J. (2005) Emotion-related processing impairments. In P.J. Eslinger (Ed.), Neuropsychological interventions: clinical research and practice (pp. 294-329). New York: Guilford.

Fleming, J., Tooth, L., Connell, J., \& Strong, J. (2002). A comparison of adjustment and self-awareness in adults after traumatic brain injury and spinal cord injury: the transition from hospital to community. Journal of Cognitive Rehabilitation, 20, 28-36.

Freed, P. (2002). Meeting of minds: Ego reintegration after traumatic brain injury. Bulletin of the Menninger Clinic, 66, 61-78.

Glisky, E.L. (2004). Disorders of memory. In J. Ponsford (Ed.), Cognitive and behavioral rehabilitation: from neurobiology to clinical practice (pp. 100-128). New York: Guilford.

High, W.M., Sander, A.M., Struchen, M.A., \& Hart, K.A. (2005). Rehabilitation for traumatic brain injury. New York: Oxford University Press.

King, N. (1997). Mild head injury: neuropathology, sequelae, measurement and recovery. British Journal of Clinical Psychology, 36, 161-184.

May, R. (1967). Psychology and the human dilemma. Princeton, N.J.: D. Van Nostrand.

May, R. (1983). The discovery of being: writing in existential psychology. New York: W.W. Norton.

Mazzucchi, A., Cattelani, R., Cavatorta, S., Parma, M., Verneri, A., \& Contini, G. (2000). Traumatic brain injury rehabilitation as an integrated task of clinicians and families: local and nationa experiences. In A.L. Christensen, \& B.P. Uzzell (Eds.) International handbook of neuropsychological rehabilitation, (pp. 299-314) New York: Kluwer Academic/Plenum.

Miller, L. (1993). Psychotherapy of the brain-injured patient: reclaiming the shattered self. New York: W.W. Norton.

Mitrushina, M., Boone, K.B., Razani, J., \& D’Elia, L.F. (2005). Handbook of normative data for neuropsychological assessment. New York: Oxford.

Moules, S., \& Chandler, B.J. (1999). A study of the health and social needs of carers of traumatically brain injured individuals served by one community rehabilitation team. Brain Injury, 13, 983-993.

Nadell, J. (1991). Towards an existential psychotherapy with the traumatically brain injured patient. Journal of Cognitive Rehabilitation, 9(6), 8-13.

Nadolne, M.J., \& Stringer, A.Y. (2001). Ecologic validity in neuropsychological assessment: prediction of wayfinding. Journal of the International Neuropsychological Society, 7, 675-682.

Ososkie, J.N. (1998). Existential perspectives in rehabilitation counseling. Rehabilitation Education, 12, 217-222.

Parker, R.S. (1990). Traumatic brain injury and neuropsychological impairment: sensorimotor, cognitive, emotional and adaptive problems of children and adults. New York: Springer-Verlag.

Prigatano, G.P. (1986). The Patient Competency Rating Scale. In G.P. Prigatano (Ed.), Neuropsychological rehabilitation after brain injury. Baltimore: Johns Hopkins University Press.

Prigatano, G.P., \& Schacter, D.L. (1991). Awareness of deficit after brain injury: clinical and theoretical issues. New York: Oxford University Press.

Prigatano, G.P. (2005). Therapy for emotional and motivational disorders. In W.M. High, Jr., A.M. Sander, M.A. Struchen, \& K.A. Hart (Eds.), Rehabilitation for traumatic brain injury (pp. 423475). New York: Oxford University Press.

Port, A., Willmott, C., \& Charlton, J. (2002). Self-awareness following traumatic brain injury and implications for rehabilitation. Brain Injury, 16, 277-289.

Rotondi, A.J., Sinkule, J., Balzer, K., Harris, J., \& Moldovan, R. (2007). A qualitative needs assessment of persons who have experienced traumatic brain injury and their primary family caregivers. Journal of Head Trauma Rehabilitation, 22(1), 14-25.

Sherer, M., Harts, T., \& Nick, T.G. (2003). Measurement of impaired self-awareness after traumatic brain injury: a comparison of the patient competency rating scale and the awareness questionnaire. Brain Injury, 17, 25-37.

Silver, J.M., Kramer, R., Greenwald, S., \& Weissman, M. (2001) The association between head injuries and psychiatric disorders: findings from the New Haven NIMH Epidemiologic Catchment Area Study. Brain Injury, 15, 935-945.

Skeed, R.L., \& Edwards, S. (2001). The assessment and rehabilitation of memory impairments. In B. Johnstone, \& H.H. Stonnington (Eds.), Rehabilitation of neuropsychological disorders: a practical guide for rehabilitation professionals (pp. 87-124). Philadelphia: Psychology Press.

Sohlberg, M.M., \& Mateer, C.A. (2001). Cognitive rehabilitation: an integrative neuropsychological approach. New York: Guilford Press.

Wade, S.L., Taylor, H.G., Drotar, D., Stancin, T., Yeates, K.O., \& Minich, N.M. (2002). A prospective study of long-term caregiver and family adaptation following brain injury in children. Journal of Head Trauma Rehabilitation, 17, 96-111.

Wilson, B.A. (1991). Theory, assessment and treatment in neuropsychological rehabilitation. Neuropsychology, 5, 281-291.

Wilson, B.A. (2002). Management and remediation of memory problems in brain-injured adults. In A.D. Baddeley, M.D. Kopelman, \& B.A. Wilson (Eds.), The handbook of memory disorders, ( 2nd edition, pp. 655-682). New York: John Wiley.

Wilson, B.A. (2003). The theory and practice of neuropsychological rehabilitation: an overview. In B.A. Wilson, \& O.L. Zangwill (Eds.), Neuropsychological rehabilitation: theory and practice, (pp. 1-10). Exton, PA: Swets \& Zeitlinger.

Yeates, K.O., Taylor, H.G., Wade, S.L., Drotar, D., Stancin, T., \& Minich, N. (2002). A prospective study of short- and long-term neuropsychological outcomes after traumatic brain injury in children. Neuropsychologia, 16, 514-523. 